\title{
Neutral oxygen irradiation enhanced forming-less ZnO-based transparent analog memristor devices for neuromorphic computing applications
}

Firman Mangasa Simanjuntak, ${ }^{1,2}$ Takeo Ohno ${ }^{3}$ Sridhar Chandrasekaran, ${ }^{4}$ Tseung-Yuen Tseng $^{5, *}$ and Seiji Samukawa ${ }^{1,6, * *}$

${ }^{1}$ World Premier Institute (WPI) - Advanced Institute for Materials Research, Tohoku University, Sendai 980-8577, Japan

${ }^{2}$ Zepler Institute of Photonics and Electronics, University of Southampton, Southampton SO17 1BJ, United Kingdom

${ }^{3}$ Department of Innovative Engineering, Oita University, Oita 870-1192, Japan

${ }^{4}$ Department of Electrical Engineering and Computer Sciences, National Chiao Tung University, Hsinchu 30010, Taiwan

${ }^{5}$ Institute of Electronics, National Chiao Tung University, Hsinchu 30010, Taiwan

${ }^{6}$ Institute of Fluid Science, Tohoku University, Sendai 980-8577, Japan

Keywords: memristor, artificial synapse, plasma irradiation, $\mathrm{ZnO}$, transparent electronics

\begin{abstract}
Surface oxidation employing neutral oxygen irradiation significantly improves the switching and synaptic performance of $\mathrm{ZnO}$-based transparent memristor devices. The endurance of the as-irradiated device is increased by 100 times, and the operating current can be lowered by 10 times as compared with the as-deposited device. Moreover, the performance-enhanced device has an excellent analog behavior that can exhibit 3-bits per cell nonvolatile multistate characteristics and perform 15 stable epochs of synaptic operations with highly linear weight updates. A simulated artificial neural network comprising 1600 synapses confirms the superiority of the enhanced device in processing a $40 \times 40$ pixels grayscale image. The irradiation effectively decreases the concentration of oxygen vacancy donor defects and promotes oxygen interstitial acceptor defects on the surface of the $\mathrm{ZnO}$ films, which consequently modulate the redox process during rupture and rejuvenation of the filament. This
\end{abstract}


work not only proposes the potential of $\mathrm{ZnO}$-based memristor devices for high-density invisible data storage and in-memory computing application but also offers valuable insight in designing high-performance memristor devices, regardless of the oxide system used, by taking advantage of our neutral oxygen irradiation technique.

$*, * *)$ Author to whom correspondence should be addressed.

Electronic mail: samukawa@ifs.tohoku.ac.jp, tseng@cc.nctu.edu.tw. 


\section{Introduction}

Data storage having analog characteristics is crucial not only for the fabrication of highdensity storage but also for the realization of in-memory computing.[1] In-memory computing is an emerging technology that could solve the memory wall issue, which will revolutionize the present computer architecture.[1,2] Memristor devices have great potential for future universal memory and can be designed in such a way as to exhibit either digital or analog characteristics.[3] The switching mechanism of the memristor relies on the electric field induced reduction and oxidation (redox) processes of defects.[4] Oxygen ions drift to the anode; meanwhile, the accumulation of oxygen vacancies that results on the cathode forms a conducting filament and switches the device on; conversely, the oxygen ions can be re-ionized and fill the vacancies to rupture the filament and switch the device off. [5]

Memristor technology, however, often suffers from several issues that hinder its application for wearable data storage and in-memory computing. First, the memristor device usually requires a forming process to activate its switching behavior, and this process might add circuit complexity in a practical application; moreover, the forming process may lead to electrical stress that causes bubble formation at the top interface and has a destructive effect on the memory cell.[6] Second, endurance degradation is a key challenge in memristor operation that limits its write-erase capability.[7-9] Third, neuromorphic computation employing memristor devices generally suffers from inefficient learning owing to its asymmetric response towards the electrical pulses.[1] Fourth, oxygen vacancy and metal interstitial are the main defects that play major role in the electronic properties in metal oxide-based devices,[10,11], however, controlling their concentration is still a great challenge, particularly for $\mathrm{ZnO}$ materials; although various methods have been proposed to achieve a high-performance transparent $\mathrm{ZnO}$-based memristor (multilayer structuring,[12-15] doping,[16-21] chemical oxidation,[3,22] deposition parameter adjustment,[23,24] electrode engineering,[25] and switching activation programming[26]), but these methods are time-consuming and complicated. 
In this letter, we propose a simple method to answer these challenges. We employ a single thin layer as the switching layer, which is sufficiently thin to achieve forming-less behavior, and oxidize the surface of the layer to mitigate the endurance decay issue. Furthermore, our enhanced devices exhibit high synaptic linearity, which is useful for in-memory computing applications.

\section{Methods}

The fabrication process is depicted in Figure 1(a). Indium tin oxide (ITO)-coated glass commercial substrates were ultrasonically-cleaned using acetone and de-ionized (DI) water before depositing 29-nm-thick $\mathrm{ZnO}$ films onto the substrates. The $\mathrm{ZnO}$ films were exposed to neutral oxygen particles for $1 \mathrm{~h}$; the plasma source power, aperture bias power, and $\mathrm{O}_{2}$ flow were $2000 \mathrm{~W}, 40 \mathrm{~W}$, and $20 \mathrm{sccm}$, respectively. This irradiation technique is also called neutral beam oxidation (NBO). The NBO system neutralizes the oxygen plasma by charge exchange collision with the inner walls of silicon aperture electrodes;[27] this technique was developed by our group to achieve highly-controlled surface chemical reactions for the fabrication of highquality nanoscale oxide films. Details of the apparatus of the NBO system have been described in our previous reports.[27-29] Aluminum-doped $\mathrm{ZnO}$ (AZO) top electrodes were patterned using a shadow mask with a diameter of $150 \mu \mathrm{m}$; the AZO was deposited in Ar ambient to achieve highly oxygen deficient conducting films. All fabrication processes were conducted at room temperature. The thickness of the top AZO and bottom ITO electrodes was found to be $275 \mathrm{~nm}$ and $95 \mathrm{~nm}$, respectively, as shown in Figure 1(b). The AZO/ZnO/ITO sandwich structure device was highly transparent (average transmittance of approximately $84 \%$ in the visible light region) and has great potential to be embedded in a touch panel and display system as well as for wearable electronic applications, as depicted in Figure 1(c). The devices or films made without and with irradiation treatment have been denoted as as-deposited and asirradiated devices/samples, respectively. 


\section{Results and discussion}

Switching characteristics of the devices were investigated by applying a voltage bias on the top electrode while the bottom electrode was ground, and the results are shown in Figure 1(e) and (e). The pristine states of the as-deposited and as-irradiated devices were found to be in the low resistance state (LRS). The left insets of Figure 1(d) and (e) depict the log (I)-log $(V)$ curves of the first positive bias employed on the pristine devices; the conduction of the pristine devices followed Ohmic behavior and the pristine resistances of the as-deposited and as-irradiated devices were calculated to be 2.1 and $5.9 \mathrm{k} \Omega$, respectively. The devices could be switched off (from the LRS to the high resistance state (HRS), also known as the reset process) by a negative bias (Vreset) of $-2.3 \mathrm{~V}$ and switched back on (from the HRS to the LRS, also known as the set process) by a positive bias (Vset) with approximately 2 and $1 \mathrm{~V}$ being required to set the as-deposited and as-irradiated devices, respectively. A current compliance (CC) of 1 and $0.1 \mathrm{~mA}$ were employed to avoid breakdown of the as-deposited and as-irradiated devices, respectively; the as-deposited device was unable to perform the set process with a $\mathrm{CC}$ of lower than $1 \mathrm{~mA}$. This indicated that the devices do not require a forming process to activate the switching behavior, which is an important characteristic to simplify the circuit design and the practical operation for application in integrated electronics.[30,31] The endurance test was carried out to assess the reliability of the devices, and the results are shown in the right insets of Figure 1(d) and (e). The as-deposited device exhibited an endurance decay after being switched for approximately 20 cycles; meanwhile, the as-irradiated device performed stable switching for more than 2000 cycles with a decent On/Off ratio of more than 1 order of magnitude. The electrical tests indicated that the as-irradiated device not only could be operated at lower operational current (by 1 order of magnitude) but also showed better endurance than the as-deposited device. Materials analysis was conducted to elucidate this phenomenon. 
It was reported that a surface treatment by plasma/ions irradiation may significantly alter the surface roughness of the films;[32,33] meanwhile, the contour of the electrode/insulator interface may affect the electric field distribution during the switching process and alter the electrical characteristics of the memristor devices.[24,34-36] However, we observed that the neutral irradiation treatment slightly affected the surface topography of the $\mathrm{ZnO}$ films; the surface roughness of the as-irradiated film was almost the same as that of the as-deposited film, as shown in Figure 2(a). Moreover, secondary-ion mass spectrometry (SIMS) analysis confirmed no etching damage on the $\mathrm{ZnO}$ films resulted from the neutral irradiation, as depicted in Figure 2(b); the ability to avoid film degradation during long irradiation is a critical advantage for device design and fabrication, which cannot be achieved by the conventional plasma technique.[32,37] This indicates that the switching enhancement may not be determined by the surface nanostructure. The plasma/ions irradiation, however, induces chemical reactions at the surface of the films.[33,38,39] We analyzed the O1s core level X-ray photoelectron spectroscopy (XPS) spectra and $\mathrm{ZnL}_{3} \mathrm{M}_{4.5} \mathrm{M}_{4.5}$ Auger lines to investigate the concentration changes of oxygen- and zinc-related defects arising from the irradiation treatment, and the results are shown in Figure 2(c) and (d), respectively. The O1s core level spectra were fitted as three Gaussian resolved component peaks; the low- $\left(\mathrm{O}_{\mathrm{I}}\right)$, medium- $\left(\mathrm{O}_{\mathrm{II}}\right)$, and high- $\left(\mathrm{O}_{\mathrm{III}}\right)$ binding energy peaks corresponded to the amount of oxygen in the fully oxidized region, deficient region (also can be referred to as oxygen vacancy $\left(V_{o}\right)$ defect), and loosely-bound oxygen (from the environment such as $\mathrm{C}-\mathrm{O}, \mathrm{OH}-$, etc., and/or oxygen interstitial $\left(\mathrm{O}_{\mathrm{i}}\right)$ defect), respectively.[40,41] Meanwhile, the $\mathrm{ZnL}_{3} \mathrm{M}_{4.5} \mathrm{M}_{4.5}$ Auger lines were fitted as two Gaussian resolved component peaks; the low- $\left(\mathrm{Zn}_{\mathrm{I}}\right)$ and high- $\left(\mathrm{Zn}_{\mathrm{II}}\right)$ binding energy peaks corresponded to the number of zinc interstitial $\left(\mathrm{Zn}_{\mathrm{i}}\right)$ defects and the amount of zinc in the oxidized region, respectively.[3] The concentrations of the $\mathrm{V}_{\mathrm{o}}$, loosely-bound oxygen and $\mathrm{Zn}_{\mathrm{i}}$ were calculated by taking the ratio of the areas of their corresponding deconvoluted components and the total area of the respective peak, and the results are shown in Figure 2(e). Generally, the $V_{o}$ 


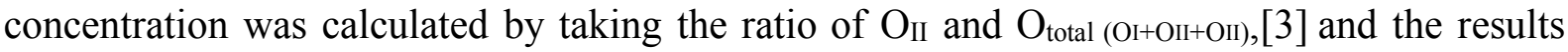
showed that the $\mathrm{V}_{\mathrm{o}}$ concentration decreased by approximately $10 \%$ after the irradiation treatment. However, if we excluded the contribution of loosely bound oxygen $\left(\mathrm{O}_{\mathrm{III}}\right)$, the decrease of the $\mathrm{V}_{\mathrm{o}}$ concentration after irradiation was only approximately $2 \%$. This indicated that the presence of loosely bound oxygen has a significant contribution to the total oxygen concentration in the lattice; the concentration of the $\mathrm{O}_{\text {III }}$ in the total components was dramatically increased by more than $14 \%$ after irradiation. The loosely-bound oxygen is commonly assumed as the absorbed oxygen from the contaminant/environment $\left(\mathrm{CO}^{-}, \mathrm{OH}^{-}\right.$, etc.).[40] However, the XPS spectra were taken after the pre-sputter condition (as approximately $1 \mathrm{~nm}$ was etched from the surface); therefore, we assumed that the loosely-bound oxygen from the contaminant/environment should have a minor contribution and the increase of the absorbed oxygen concentration should result from irradiation. This absorbed oxygen interstitially exists in the lattice and can be identified as $\mathrm{O}_{i}$ defects.[41] Meanwhile, the irradiation had no effect on modulating the concentration of $\mathrm{Zn}_{\mathrm{i}}$ defect, as shown in Figure 2(e). Therefore, we were able to suggest that the enrichment of irradiation-induced $\mathrm{O}_{\mathrm{i}}$ defects on the surface of the $\mathrm{ZnO}$ switching layer contributes to the performance enhancement in the memristor operation.

During the first switching (reset process) of the as-deposited device, the high reset current (approximately $1 \mathrm{~mA}$ ) indicating a large size of filaments were initially formed due to the high number of oxygen vacancies in the film. Meanwhile, for the as-irradiated device, abundant amount of $\mathrm{O}_{\mathrm{i}}$ and lattice oxygens (located at the $\mathrm{AZO} / \mathrm{ZnO}$ interface) can be ionized and fill a large portion of the pristinely-formed oxygen vacancy filaments; note that, the nature of the $\mathrm{O}_{\mathrm{i}}$ defects can easily drift under an electric field which is beneficial to accelerate the rupture process.[9] Since the highly oxidized surface contains abundant $\mathrm{O}_{\mathrm{i}}$ acceptor defects, it contributes to limit the high number of electrons that can be injected from the top electrode; consequently, the reset process exhibits a lower reset current (approximately $0.1 \mathrm{~mA}$ ) compared 
with that of the as-deposited device (approximately $1 \mathrm{~mA}$ ) (Figure 1(d) and (e)). The filament in the as-irradiated device can then be rejuvenated by employing a lower CC and Vset $(0.1 \mathrm{~mA}$ and $\sim 1 \mathrm{~V}$, respectively, as shown in Figure 1(e)), whereas the set process in the as-deposited device requires a higher $\mathrm{CC}$ and set voltage ( $1 \mathrm{~mA}$ and $\sim 2 \mathrm{~V}$, respectively, Figure 1(d)) since the as-deposited device has a higher leakage current arising from the abundant amount of donor defects. In addition to the high switching parameter ( $\mathrm{CC}$ and Vset), oxygen in-diffusion to the top electrode also leads to an ion-vacancy imbalance that results in endurance degradation.[79] We can assume that the as-deposited device had a thicker conduction filament owing to the high $\mathrm{CC}$ and Vset; thus, a higher number of oxygen ions was needed to rupture a significant portion of the filament. Meanwhile, the number of oxygen ions at the $\mathrm{AZO} / \mathrm{ZnO}$ interface decreased at each set process owing to the oxygen in-diffusion to the highly oxygen-deficient AZO electrode. Consequently, the as-deposited device exhibited endurance degradation. However, the as-irradiated device had a thinner conduction filament (owing to the low CC and Vset), and a higher concentration of oxygen at the $\mathrm{AZO} / \mathrm{ZnO}$ interface maintained the ionvacancy balance at each switching process; thus, the as-irradiated device exhibited better endurance. Table 1 lists the various methods and device designs that have been proposed to fabricate $\mathrm{ZnO}$-based transparent memristor devices. By considering the thickness as well as the operational switching current and voltages, we can infer that our memristor device consumes less power than those of the other designs/methods.

Our memristor device exhibited gradual set and reset processes, which indicated that the switching occurs in analog behavior. A memristor having analog behavior is crucial for the realization of high-density data storage and in-memory computing (neuromorphic) systems.[1] Figure 3(a) demonstrates the ability of the as-irradiated device to exhibit a multistate characteristic employing various Vreset, and each of the states showed sufficient non-volatility retention; the device performed 3-bits per cell (a total of 8 states), which indicated that the $\mathrm{AZO} / \mathrm{ZnO} / \mathrm{ITO}$ device structure can be useful for the fabrication of high-density storage. We 
then explored the potential of our devices for mimicking biological synaptic plasticity. Figure 3(b) depicts the schematic of the communication between two nerve cells (neurons); information transmission is controlled by a firing mechanism of $\mathrm{Ca}^{2+}$ ions (transmitters) from presynaptic to postsynaptic neurons that are located at the synapse (the junction between cells) and results in the modulation of synaptic weight.[2] Similarly, an analog memristor can also be exploited to make an artificial synapse that exhibits such synaptic weight modulation by "firing" it with voltage-pulse spikes to fine-tune the ion-vacancy redox reactions. The technical details regarding the concept of synaptic plasticity can also be found in previous reports.[2,3,23,42-46] The increase and decrease of synaptic weight can be referred to as potentiation and depression, respectively; each of the electrical schemes for the potentiation and depression was repeated for 1000 times, as shown in Figure 3(c). The as-irradiated device exhibited 15 stable epoch training, as depicted in Figure 3(d); an epoch consisted of a potentiation and a depression with a total of 2000 spikes (inset of Figure 3(d)). Moreover, the as-irradiated device showed excellent weight update symmetry with a nonlinearity of $18 \%$, as depicted in Figure 3(e). For comparison, we also measured the synaptic weight update of the as-deposited device, as shown in Figure 3(f). Although the device was also able to show stable epoch training, the device suffered from weight update asymmetry with a nonlinearity of $63 \%$, as shown in Figure 3(g). The poor synaptic linearity of the as-deposited device may have arisen from two factors. First, the high imbalance of ion-vacancies and high operational current. The conductance changed rapidly at the initial potentiation since it was easy to generate an oxygen vacancy or rejuvenate the filament, and once the filament formed, the conductance became saturated; meanwhile, the high operational current during the depression process re-ionized a significant amount of oxygen to fill the vacancies and the conductance started to saturate once the oxygen from the top interface was about to be depleted. The good ion-vacancy balance and low operating current of the as-irradiated device were beneficial to achieve excellent synaptic linearity. Second, it is reported that the high concentration of oxygen at certain level decreases 
the thermal conductivity of $\mathrm{ZnO}$ film, [47] that can be assumed the generation of high $\mathrm{O}_{\mathrm{i}}$ concentration may induce phonon scattering as well; therefore, the as-deposited film may have a high thermal conductivity than that of as-irradiated one. The higher thermal conductivity may further promote the formation of strong filaments that degenerate synaptic linearity.[48] Synaptic linearity is a crucial factor in the implementation of artificial neural networks (ANN). Based on the experimental synaptic data, we assessed the feasibility of our devices for neuromorphic computing by simulating an array level of 1600 neurons to mimic the visual cortex of the brain, as depicted in Figure 3(h). The synaptic data were fed to the network to process a $40 \times 40$ pixels grayscale image; the network was built by adopting Hopefield's method that has been described in our previous report.[45] The as-irradiated device was able to process the image much faster than the as-deposited device; the as-irradiated device could achieve $90 \%$ accuracy after 18 iterations while the as-deposited device could only achieve $60 \%$ accuracy, as depicted in Figure 3(i). This arose from the high nonlinearity of the as-deposited device, which causes a training accuracy loss in neural networks.[1]

\section{Conclusions}

The switching and synaptic performances of $\mathrm{ZnO}$-based transparent memristor devices are significantly enhanced after neutral oxygen irradiation. The employment of neutral oxygen particles is crucial to achieve precise surface chemical oxidation to avoid abnormal etching and surface roughness degradation. The surface oxidation increases the oxygen interstitial concentration at the top electrode/ $\mathrm{ZnO}$ interface that is beneficial not only to improve switching parameters (low current compliance and set voltage, and long endurance) but also to improve the synaptic weight linearity. The artificial neural network simulation based on the synaptic data of the as-irradiated device shows better training accuracy during image processing. This study suggests the potential use of neutral beam oxidation enhanced $\mathrm{ZnO}$-based memristor not only for fabricating high-density invisible data storage but also for the realization of wearable 
in-memory computing. Moreover, our surface oxidation method can be easily adopted and explored for use in other oxide memristor designs. It worth noting that this surface modification technique could be further exploited to control other synaptic parameters such as long-/shortterm memory, spike-timing-dependent plasticity, etc.

\section{Acknowledgments}

The authors acknowledge experimental support from Mr. Kesami Saito for film deposition and atomic force microscopy characterization and Mr. Toshiya Kojima for SIMS characterization. The authors thank the financial supports of the AIMR, Tohoku University, sponsored by the WPI program, MEXT, Japan and the National Chiao Tung University, Taiwan.

\section{References}

[1] Yu S 2018 Neuro-Inspired Computing with Emerging Nonvolatile Memorys Proc. IEEE $106260-85$

[2] Ohno T, Hasegawa T, Tsuruoka T, Terabe K, Gimzewski J K and Aono M 2011 Short-term plasticity and long-term potentiation mimicked in single inorganic synapses Nat. Mater. 10 591-5

[3] Simanjuntak F M, Chandrasekaran S, Lin C and Tseng T-Y 2019 ZnO2/ZnO bilayer switching film for making fully transparent analog memristor devices APL Mater. 7 051108

[4] Aluguri R, Kumar D, Simanjuntak F M and Tseng T-Y 2017 One bipolar transistor selector - One resistive random access memory device for cross bar memory array AIP $A d v .7095118$

[5] Chandrasekaran S, Simanjuntak F M, Aluguri R and Tseng T 2018 The impact of TiW 
barrier layer thickness dependent transition from electro-chemical metallization memory to valence change memory in $\mathrm{ZrO} 2$-based resistive switching random access memory devices Thin Solid Films $660777-81$

[6] Huang J-J, Kuo C-W, Chang W-C and Hou T-H 2010 Transition of stable rectification to resistive-switching in $\mathrm{Ti} / \mathrm{TiO}[\mathrm{sub} 2] / \mathrm{Pt}$ oxide diode Appl. Phys. Lett. 96262901

[7] Raghavan N, Pey K L, Frey D D and Bosman M 2014 Stochastic failure model for endurance degradation in vacancy modulated HfOx RRAM using the percolation cell framework IEEE Int. Reliab. Phys. Symp. Proc. 1-7

[8] Lu Y, Chen B, Gao B, Fang Z, Fu Y H, Yang J Q, Liu L F, Liu X Y, Yu H Y and Kang J F 2012 Improvement of endurance degradation for oxide based resistive switching memory devices correlated with oxygen vacancy accumulation effect 2012 IEEE Int. Reliab. Phys. Symp. MY.4.1-MY.4.4

[9] Chen C Y, Goux L, Fantini A, Clima S, Degraeve R, Redolfi A, Chen Y Y, Groeseneken G and Jurczak M 2015 Endurance degradation mechanisms in TiN\Ta2O5\Ta resistive random-access memory cells Appl. Phys. Lett. 106

[10] Qin T, Zhang X, Wang D, Deng T, Wang H, Liu X, Shi X, Li Z, Chen H, Meng X, Zhang W and Zheng W 2019 Oxygen Vacancies Boost $\delta$-Bi 2 O 3 as a HighPerformance Electrode for Rechargeable Aqueous Batteries ACS Appl. Mater. Interfaces $112103-11$

[11] Labégorre J B, Lebedev O I, Bourgès C, Rečnik A, Košir M, Bernik S, Maignan A, Le Mercier T, Pautrot-D’Alençon L and Guilmeau E 2018 Phonon Scattering and Electron Doping by 2D Structural Defects in In/ZnO ACS Appl. Mater. Interfaces 10 6415-23

[12] Zheng K, Sun X W, Zhao J L, Wang Y, Yu H Y, Demir H V. and Teo K L 2011 An 
Indium-Free Transparent Resistive Switching Random Access Memory IEEE Electron Device Lett. 32 797-9

[13] Chun-Yang Huang, Yen-Ting Ho, Chung-Jung Hung and Tseung-Yuen Tseng 2014 Compact Ga-Doped ZnO Nanorod Thin Film for Making High-Performance Transparent Resistive Switching Memory IEEE Trans. Electron Devices 61 3435-41

[14] Yang P-K, Chang W-Y, Teng P-Y, Jeng S-F, Lin S-J, Chiu P-W and He J-H 2013 Fully Transparent Resistive Memory Employing Graphene Electrodes for Eliminating Undesired Surface Effects Proc. IEEE 101 1732-9

[15] Zhang R, Miao J, Shao F, Huang W T, Dong C, Xu X G and Jiang Y 2014 Transparent amorphous memory cell: A bipolar resistive switching in $\mathrm{ZnO} / \mathrm{Pr} 0.7 \mathrm{Ca} 0.3 \mathrm{MnO} 3 / \mathrm{ITO}$ for invisible electronics application J. Non. Cryst. Solids 406 102-6

[16] Shi L, Shang D, Sun J and Shen B 2009 Bipolar Resistance Switching in Fully Transparent ZnO:Mg-Based Devices Appl. Phys. Express 2101602

[17] Cao X, Li X, Gao X, Liu X, Yang C, Yang R and Jin P 2011 All-ZnO-based transparent resistance random access memory device fully fabricated at room temperature J. Phys. D. Appl. Phys. 44255104

[18] Yu H, Kim M, Kim Y, Lee J, Kim K, Choi S and Cho S 2014 Al-doped ZnO as a switching layer for transparent bipolar resistive switching memory Electron. Mater. Lett. $10321-4$

[19] Simanjuntak F M, Prasad O K, Panda D, Lin C-A, Tsai T-L, Wei K-H and Tseng T-Y 2016 Impacts of Co doping on $\mathrm{ZnO}$ transparent switching memory device characteristics Appl. Phys. Lett. 108183506

[20] Kim A, Song K, Kim Y and Moon J 2011 All Solution-Processed, Fully Transparent 
Resistive Memory Devices ACS Appl. Mater. Interfaces 3 4525-30

[21] Chen M-C, Chang T-C, Huang S-Y, Chen S-C, Hu C-W, Tsai C-T and Sze S M 2010 Bipolar Resistive Switching Characteristics of Transparent Indium Gallium Zinc Oxide Resistive Random Access Memory Electrochem. Solid-State Lett. 13 H191

[22] Simanjuntak F M, Pattanayak B, Lin C-C and Tseng T-Y 2017 Resistive Switching Characteristics of Hydrogen Peroxide Surface Oxidized ZnO-Based Transparent Resistive Memory Devices ECS Trans. 77 155-60

[23] Simanjuntak F M, Ohno T and Samukawa S 2019 Film-Nanostructure-Controlled Inerasable-to-Erasable Switching Transition in ZnO-Based Transparent Memristor Devices: Sputtering-Pressure Dependency ACS Appl. Electron. Mater. $12184-9$

[24] Simanjuntak F M, Ohno T and Samukawa S 2019 Influence of rf sputter power on ZnO film characteristics for transparent memristor devices AIP Adv. 9105216

[25] Simanjuntak F M, Panda D, Tsai T-L, Lin C-A, Wei K-H and Tseng T-Y 2015 Enhancing the memory window of $\mathrm{AZO} / \mathrm{ZnO} / \mathrm{ITO}$ transparent resistive switching devices by modulating the oxygen vacancy concentration of the top electrode $J$. Mater. Sci. 50 6961-9

[26] Simanjuntak F M, Panda D, Tsai T-L, Lin C-A, Wei K-H and Tseng T-Y 2015 Enhanced switching uniformity in $\mathrm{AZO} / \mathrm{ZnO} 1-\mathrm{x} / \mathrm{ITO}$ transparent resistive memory devices by bipolar double forming Appl. Phys. Lett. 107033505

[27] Ohno T, Nakayama D, Okada T and Samukawa S 2018 Energy control of neutral oxygen particles passing through an aperture electrode Results Phys. 8 169-71

[28] Ohno T and Samukawa S 2015 Resistive switching in a few nanometers thick tantalum oxide film formed by a metal oxidation Appl. Phys. Lett. 106173110 
[29] Ohno T, Nakayama D and Samukawa S 2015 Al and Ge simultaneous oxidation using neutral beam post-oxidation for formation of gate stack structures Appl. Phys. Lett. 107 133107

[30] Kurnia F, Liu C, Jung C U and Lee B W 2013 The evolution of conducting filaments in forming-free resistive switching $\mathrm{Pt} / \mathrm{TaO}$ x /Pt structures Appl. Phys. Lett. 102 152902

[31] Mao Q, Ji Z and Xi J 2010 Realization of forming-free ZnO-based resistive switching memory by controlling film thickness J. Phys. D. Appl. Phys. 43395104

[32] Meena J S, Chu M-C, Chang Y-C, You H-C, Singh R, Liu P-T, Shieh H-P D, Chang F-C and Ko F-H 2013 Effect of oxygen plasma on the surface states of $\mathrm{ZnO}$ films used to produce thin-film transistors on soft plastic sheets J. Mater. Chem. C 16613

[33] Huang T-H, Yang P-K, Lien D-H, Kang C-F, Tsai M-L, Chueh Y-L and He J-H 2014 Resistive Memory for Harsh Electronics: Immunity to Surface Effect and High Corrosion Resistance via Surface Modification Sci. Rep. 44402

[34] Jeong H Y, Kim Y I, Lee J Y and Choi S-Y 2010 A low-temperature-grown TiO2based device for the flexible stacked RRAM application. Nanotechnology 21115203

[35] Nandi S K, Liu X, Venkatachalam D K and Elliman R G 2015 Effect of Electrode Roughness on Electroforming in $\mathrm{HfO} 2$ and Defect-Induced Moderation of ElectricField Enhancement Phys. Rev. Appl. 4064010

[36] Xue W H, Xiao W, Shang J, Chen X X, Zhu X J, Pan L, Tan H W, Zhang W B, Ji Z H, Liu G, Xu X-H, Ding J and Li R-W 2014 Intrinsic and interfacial effect of electrode metals on the resistive switching behaviors of zinc oxide films Nanotechnology $\mathbf{2 5}$ 425204 
[37] Chu M-C, Meena J S, Liu P-T, Shieh H-P D, You H-C, Tu Y-W, Chang F-C and Ko F-H 2013 Oxygen Plasma Functioning of Charge Carrier Density in Zinc Oxide ThinFilm Transistors Appl. Phys. Express 676501

[38] Chen P-H, Chang T-C, Chang K-C, Tsai T-M, Pan C-H, Chen M-C, Su Y-T, Lin C-Y, Tseng Y-T, Huang H-C, Wu H, Deng N, Qian H and Sze S M 2017 Resistance Switching Characteristics Induced by O 2 Plasma Treatment of an Indium Tin Oxide Film for Use as an Insulator in Resistive Random Access Memory ACS Appl. Mater. Interfaces 9 3149-55

[39] Lai Y, Zeng Z, Liao C, Cheng S, Yu J, Zheng Q and Lin P 2016 Ultralow switching current in $\mathrm{HfO} \times \mathrm{x} / \mathrm{ZnO}$ bilayer with tunable switching power enabled by plasma treatment Appl. Phys. Lett. 109063501

[40] Simanjuntak F M, Ohno T and Samukawa S 2019 Neutral Oxygen Beam Treated ZnO-Based Resistive Switching Memory Device ACS Appl. Electron. Mater. 1 18-24

[41] Fan Hai-Bo Y S-Y, Pan-Feng Z and Wei Hong-Yuan E al. 2007 Investigation of Oxygen Vacancy and Interstitial Oxygen defects in ZnO films by PL and XPS Chinese Phys. Lett. 242108

[42] Jo S H, Chang T, Ebong I, Bhadviya B B, Mazumder P and Lu W 2010 Nanoscale memristor device as synapse in neuromorphic systems Nano Lett. 10 1297-301

[43] Nayak A, Ohno T, Tsuruoka T, Terabe K, Hasegawa T, Gimzewski J K and Aono M 2012 Controlling the synaptic plasticity of a $\mathrm{Cu} 2 \mathrm{~S}$ gap-type atomic switch Adv. Funct. Mater. 22 3606-13

[44] Milano G, Luebben M, Ma Z, Dunin-Borkowski R, Boarino L, Pirri C F, Waser R, Ricciardi C and Valov I 2018 Self-limited single nanowire systems combining all-in- 
one memristive and neuromorphic functionalities Nat. Commun. 9 1-10

[45] Chandrasekaran S, Simanjuntak F, Saminathan R, Panda D and Tseng T-Y 2019 Improving linearity by introducing $\mathrm{Al}$ in $\mathrm{HfO} 2$ as memristor synapse device Nanotechnology 6 107-13

[46] Chandrasekaran S, Simanjuntak F M, Panda D and Tseng T-Y 2019 Enhanced Synaptic Linearity in ZnO-Based Invisible Memristive Synapse by Introducing Double Pulsing Scheme IEEE Trans. Electron Devices 1-5

[47] Xu Y, Goto M, Kato R, Tanaka Y and Kagawa Y 2012 Thermal conductivity of ZnO thin film produced by reactive sputtering J. Appl. Phys. 111

[48] Wu W, Wu H, Gao B, Yao P, Zhang X, Peng X, Yu S and Qian H 2018 A methodology to improve linearity of analog RRAM for neuromorphic computing Digest of Technical Papers - Symposium on VLSI Technology 


\section{Figure captions}

Figure 1. (a) Schematic of device fabrication process flow. (b) Cross-sectional transmission electron microscopy (TEM) image of AZO/ZnO/ITO/glass device structure. (c) Transmittance spectra of the fabricated devices. Inset in (c) shows the photograph of the fabricated devices. Typical I-V curves of (d) as-deposited and (e) as-irradiated devices. The left insets in (d) and (e) show the $\log (\mathrm{I})-\log (\mathrm{V})$ curves taken from the first positive bias on the pristine devices, while the right insets show the endurance characteristics of the devices.

Figure 2. (a) Surface topographies of the as-deposited and as-irradiated devices. (b) SIMS profiles of the $\mathrm{ZnO} / \mathrm{ITO}$ structure before and after irradiation. (c) X-ray photoelectron spectra of O1s core level and (d) Auger lines of $\mathrm{ZnL}_{3} \mathrm{M}_{4.5} \mathrm{M}_{4.5}$ of the $\mathrm{ZnO}$ films. (e) Defect concentrations in the films that are extracted from (c) and (d).

Figure 3. (a) Multistate characteristics of the as-irradiated device. (b) Schematic of nerve cells, an organic synapse, and a memristor cell as an inorganic artificial synapse. (c) Electrical spike schemes for potentiation and depression; the spike event consists of a pulse amplitude of $1.5 \mathrm{~V}$ (or $-1.5 \mathrm{~V}$ for depression) and a pulse width of $0.1 \mathrm{~ms}$, and the read event consists of a pulse amplitude of $0.1 \mathrm{~V}$ and a pulse width of $1 \mathrm{~ms}$. (d) Training epochs characteristics and (e) synaptic linearity of the as-irradiated device. (f) Training epochs characteristics and (g) synaptic linearity of the as-deposited device. (h) Artificial neural network simulation on image processing of $40 \times 40$ pixels grayscale patterns. 


\section{Table captions}

Table 1. Switching parameters of $\mathrm{ZnO}$-based transparent memristor devices in the published literature. 
a)
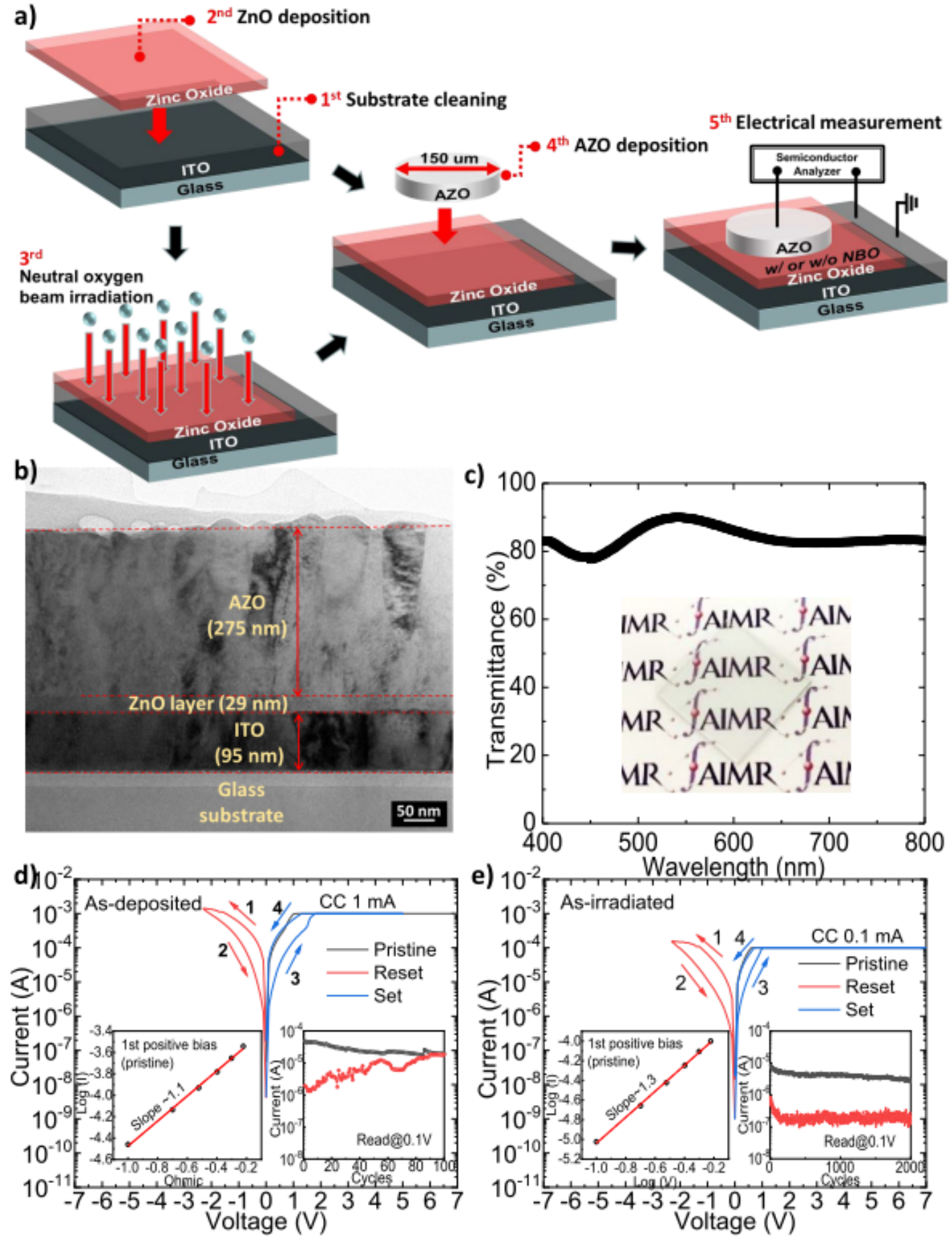

Fig. 1 Simanjuntak et. al. 

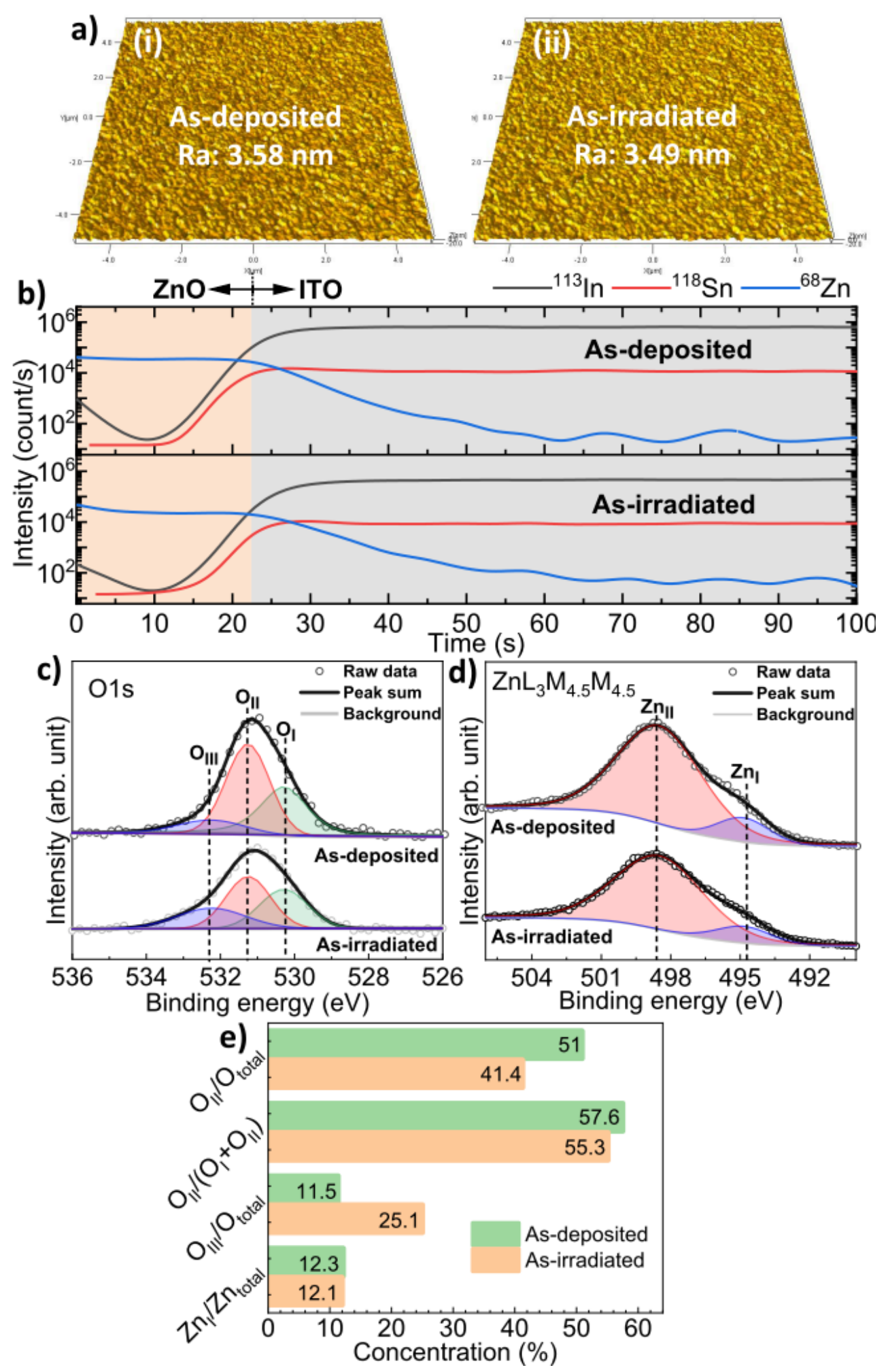

Fig. 2 Simanjuntak et. al. 


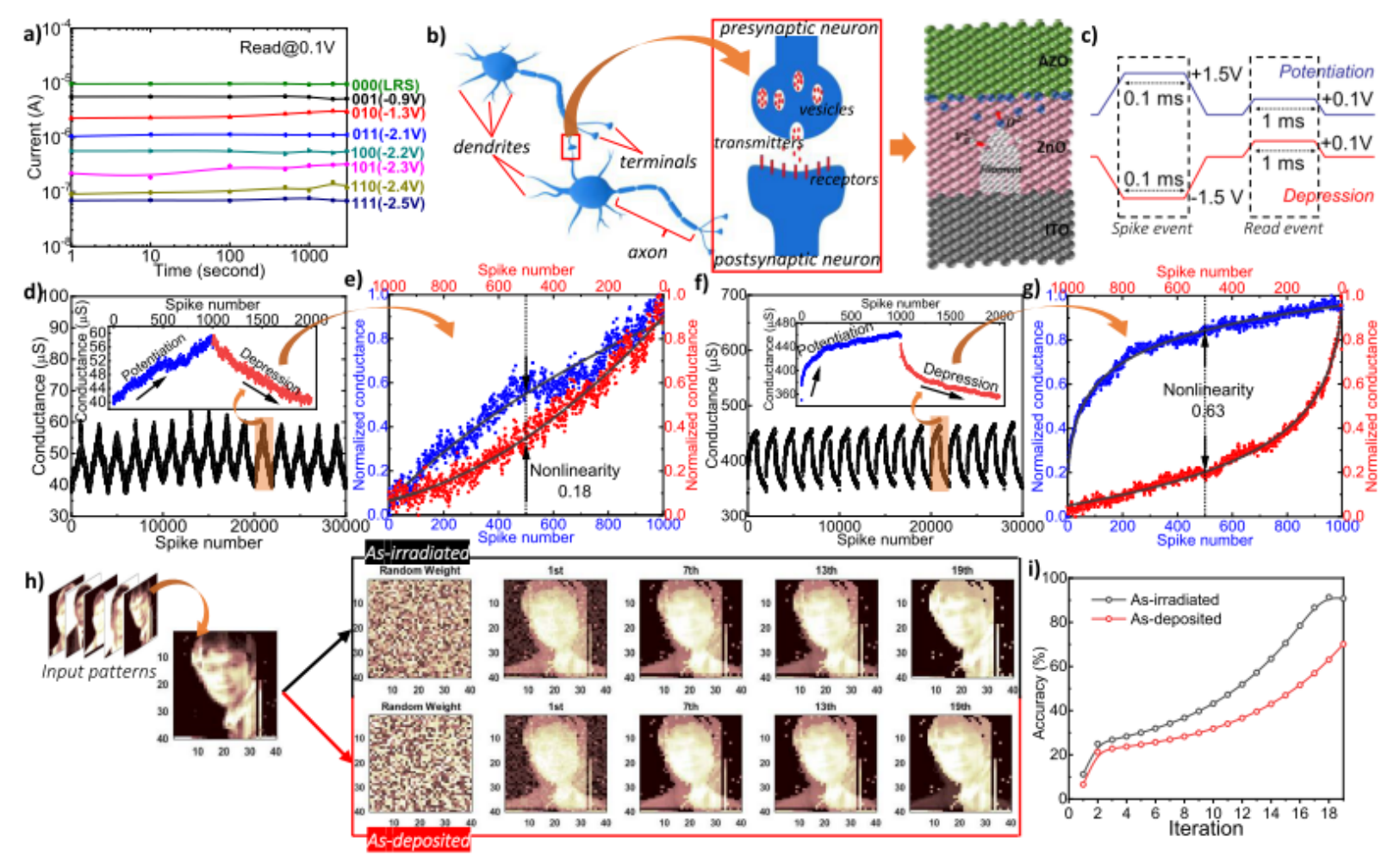

Fig. 3 Simanjuntak et. al. 


\begin{tabular}{|c|c|c|c|c|c|c|c|c|}
\hline No & Structure & $\begin{array}{c}d \\
{[\mathrm{~nm}]}\end{array}$ & $\begin{array}{c}\mathbf{T} \\
{[\%]}\end{array}$ & $\begin{array}{c}\mathbf{C C} \\
{[\mathbf{m A}]}\end{array}$ & $\begin{array}{c}\mathbf{V}_{\mathbf{F}} \\
{[\mathbf{V}]}\end{array}$ & $\begin{array}{l}V_{R} \\
{[V]}\end{array}$ & $\begin{array}{c}\mathbf{V}_{\mathbf{S}} \\
{[\mathbf{V}]}\end{array}$ & Ref. \\
\hline 1 & $\mathrm{GZO} / \mathrm{Ga}_{2} \mathrm{O}_{3} / \mathrm{ZnO} / \mathrm{Ga}_{2} \mathrm{O}_{3} / \mathrm{GZO}$ & 220 & 92 & 20 & FL & -12 & 14 & {$[12]$} \\
\hline 2 & ITO/GZO-nanorods/ZnO/ITO & 250 & $\sim 80$ & 10 & $\sim 3$ & $\sim(-2)$ & $\sim 2$ & [13] \\
\hline 3 & ITO/graphene/ZnO/ITO & 50 & 75.6 & 5 & 4 & $\sim(-2.5)$ & $\sim 1$ & [14] \\
\hline 4 & ITO/ZnO/PCMO/ITO & 160 & 79.6 & 10 & FL & $\sim 2.3$ & $\sim(-2.6)$ & {$[15]$} \\
\hline 5 & $\mathrm{GZO} / \mathrm{ZnO}_{2} / \mathrm{ZnO} / \mathrm{ITO}$ & 54 & 87.4 & 1 & $\sim 5.5$ & -1.7 & $\sim 1.5$ & [3] \\
\hline 6 & ITO/ZnO:Mg/FTO & 300 & $\sim 80$ & 50 & 2.8 & -3 & 1.8 & [16] \\
\hline 7 & $\mathrm{AZO} / \mathrm{ZnO}: \mathrm{Mg} / \mathrm{AZO}$ & 120 & $\sim 73$ & 1 & -6 & $\sim(-4)$ & $\sim 3$ & [17] \\
\hline 8 & ITO/ZnO:Al/ITO & 110 & $\sim 80$ & 10 & $\sim 2.3$ & $\sim(-0.5)$ & $\sim 0.5$ & [18] \\
\hline 9 & ITO/ZnO:Co/ITO & 38 & $\sim 85$ & 5 & 3 & -1.5 & 1.2 & [19] \\
\hline 10 & ITO/ZnO:Ga/ITO & $\sim 30$ & 86.5 & 0.1 & FL & -7 & $\sim 5$ & [20] \\
\hline 11 & ITO/ZnO:In:Ga/ITO & 36 & $\sim 75$ & 10 & FL & $\sim 3.5$ & $\sim(-1)$ & [21] \\
\hline 12 & ITO/ZnO/ITO & 80 & 88 & 5 & 2.7 & -2.4 & 1.6 & {$[46]$} \\
\hline 13 & $\mathrm{AZO} / \mathrm{ZnO}_{1-x} / \mathrm{ITO}$ & 53 & $\sim 85$ & 1 & $-5.5 / 4$ & -2 & $\sim 1.7$ & [26] \\
\hline 14 & $\mathrm{AZO} /(\mathrm{NBO}) \mathrm{ZnO} / \mathrm{ITO}$ & 29 & $\sim 84$ & 0.1 & FL & -2.3 & $\sim 1$ & This work \\
\hline
\end{tabular}

The $d, \mathrm{~T}, \mathrm{CC}, \mathrm{V}_{\mathrm{F}}, \mathrm{V}_{\mathrm{R}}, \mathrm{V}_{\mathrm{S}}$, and $\mathrm{FL}$ are the thickness of the switching layer, average transmittance in the visible light region, compliance current, as well as forming (or FL: forming-less), reset and set voltages, respectively.

Table 1. Simanjuntak et al. 